\title{
Extraction and analysis of micro-Doppler signatures by the Empirical Mode Decomposition
}

\author{
Amy Brewster *,+ \\ ${ }^{+}$Royal Australian Air Force \\ Joint Electronic Warfare Operational Support Unit \\ Adelaide, Australia
}

\author{
Alessio Balleri * \\ ${ }^{*}$ Centre for Electronic Warfare \\ Cranfield University \\ Defence Academy of the UK \\ Shrivenham, UK \\ Email: a.balleri@cranfield.ac.uk
}

\begin{abstract}
A set of experimental trials was conducted with a 10 GHz continuous wave radar to collect micro-Doppler signatures of a large single, double and triple bladed rotating fin and a sized miniature helicopter. We analysed the target microDoppler signatures and decomposed them using the Empirical Mode Decomposition (EMD) method in order to extract a series of Intrinsic Mode Functions (IMFs) which admit only an instantaneous frequency. The aim of this paper is to investigate what information is available in the target IMFs to help identify key features that can be used for improving target classification and identification. The experimental testing was complimented with a set of simulations to assist in the understanding of the results.
\end{abstract}

\section{INTRODUCTION}

The ability to classify and identify moving ground and aerial targets in order to assist in surveillance, combat situations and monitoring has recently become a topic of great interest. Moving targets can be classified with a Doppler radar system that is capable of detecting small variations in the Doppler frequency, called micro-Doppler. Micro-Doppler signatures are frequency modulations induced by small movements within the target, such as vibration and rotation. An example of micro Doppler signature is the frequency modulation induced by the rotating blades of a helicopter. Specific velocities and blade rotational speeds result in a unique micro-Doppler signature that can be used to classify the target.

Previous work to classify targets by their micro-Doppler signatures have typically used the Short Time Fourier Transform (STFT) although this imposes a limitation in terms of frequency-time resolution; the longer the dwell time on the target the better the frequency resolution and vice versa. MicroDoppler signatures and the STFT have been used together to classify many types of target including humans, animals, cars, tanks, helicopters and jet engines [1][2][3][4]. A study of the micro-Doppler signature of helicopters and a method to extract key target features was presented in [5]. Target classification of aircraft by their micro-Doppler modulations was presented in [6] and [7]. In [8], the authors derived the Maximum Likelihood (ML) estimators and their relative Cramér-Rao bounds to estimate the number of blades of a helicopter, their length and their velocity. Previous research has also shown that it is possible to generate radar images of the helicopter rotating blades with the use of tomography [9][10][11].

The Empirical Mode Decomposition (EMD) is a method to decompose target echoes into a series of Intrinsic Mode
Functions (IMFs) which does not suffer from frequency time resolution problems and hence can potentially provide more robust features and ultimately lead to improved and more robust classification performance.

The first article on EMD was published in 1998 [12]. The use of the EMD together with the Hilbert Huang Transform (HHT) for the analysis of human micro-Doppler signatures was introduced in [13]. In this paper the authors carried out a set of simulations to generate the micro-Doppler signatures of a human target swinging the arms and breathing. They showed that, as expected, the HHT applied to the simulated data resulted in a better visual resolution than the STFT. They corroborated their results on a set of experimental data collected in L band for a walking human and also showed that the main IMFs of a breathing human had different characteristics from those obtained when no human was present in the scene. The EMD and micro-Doppler signatures were used together to classify human motions in [14]. In this paper the authors investigated the use of EMD as a method to extract features of human movement for target classification. The results from a set of simulations of simple human movement were presented together with those from experimental data in S-band and W-band (millimetre wave) collected with two noncoherent Continuous Wave (CW) radars. The energy of each IMF component was calculated and used as a feature vector in a Support Vector Machines classifier. The authors showed that classification performance of human motions in a throughthe-wall indoor environment could reach an accuracy of up to $75 \%$ and that the mm-wave radar was capable of classifying the same motions with an accuracy of up to $80-90 \%$. Similar results for a radar operating at $750 \mathrm{MHz}$ were presented in [15] and [16] and for a Noise Radar for through-the-wall imaging in [17]. In [18], the authors presented an algorithm to improve the Hilbert-Huang spectrum based on a weighted average of the micro-Doppler frequency components for each IMF.

There has been very limited work in the literature on EMD and micro-Doppler and little of the published work in this area is with experimental data. To the best of our knowledge, there is very little work in the open literature carried out on the EMD applied to targets carrying rotating blades and no prior work on real data at X-band.

In this paper, we extract the IMFs from echoes of a windmill like target and a small helicopter in order to investigate the information available in the IMFs and their physical significance. The aim of the paper is to identify potential new features that may improve automatic target classification. A set 
of experimental trials were conducted with a $10 \mathrm{GHz} \mathrm{CW}$ radar to collect micro-Doppler signatures for a large single, double and triple bladed rotating fin as well as a sized miniature helicopter. The experimental testing was complimented with a set of simulations to assist in the interpretation of the results.

\section{THE EMPIRICAL MODE DECOMPOSITION}

The EMD is a method that decomposes a signal into a series of Intrinsic Mode Functions (IMFs) which admit only one instantaneous frequency at each time. An IMF is defined as a function that satisfies two essential criteria: 1) the total number of extrema (maximum and minimums) and the total number of zero crossing points must be equal or differ of no more than one and 2) the mean value between the two envelopes identified by the local maxima and the local minima must be zero at any time. The IMFs are obtained from the target echo signal $x(n)$ with a procedure that takes the name of sifting process. This involves finding all the maximum and the minimum extrema of the echo signal and then use a cubic spline function to interpolate between the maximum extrema and the minimum extrema to form an upper envelope and a lower envelope, respectively. Once the envelopes are established, the mean value $m_{i}$ between the two envelopes is calculated and subtracted from the original signal to generate a new signal $h_{i}(n)$ as

$$
x(n)-m_{i}(n)=h_{i}(n)
$$

This process is iteratively repeated on $h_{i}(n)$ until all the maxima extrema are positive and all the minimum extrema are negative, and the residue signal $h_{i}(n)$ is an IMF. Because the condition on the mean value between the upper and lower envelopes being zero is difficult to satisfy, in practice the algorithm stops when the quantity

$$
\sum_{n=1}^{N} \frac{\left(h_{i(k-1)}(n)-h_{i(k)}(n)\right)^{2}}{h_{i(k)}(n)^{2}}
$$

falls below a predefined threshold $\gamma$. It was experimentally observed that a suitable number for the threshold was $\gamma=$ 0.3 . Once this alternative condition is satisfied then $h_{i}(k)$ is designated as an IMF. The IMF is then subtracted from the original signal and the process is repeated, until the residue becomes a monotonic function.

\section{Simulation Results}

A set of simulations were carried out in order to better understand the expected level of information available in the IMFs. Echoes were simulated for a one-point-target single bladed windmill rotating at an angular rotation speed of 300 RPM (Rotations Per Minute) corresponding to a rotation frequency of $5 \mathrm{~Hz}$. The windmill was simulated with a blade length of $2.5 \mathrm{~m}$ and at a distance of $20 \mathrm{~km}$ from a $10 \mathrm{GHz}$ $\mathrm{CW}$ radar. The simulation parameters resulted in a maximum blade velocity of $78.5 \mathrm{~m} / \mathrm{s}$, which corresponds to a maximum Doppler shift of $5233.3 \mathrm{~Hz}$. The EMD algorithm was applied to the in-phase component of the simulated echoes in order to extract and analyse the resulting IMFs. A 2-second long echo signal was simulated in order to allow the view of multiple cycles of the micro-Doppler signature. The results of the simulations are shown in Figure 1 and Figure 2. Results

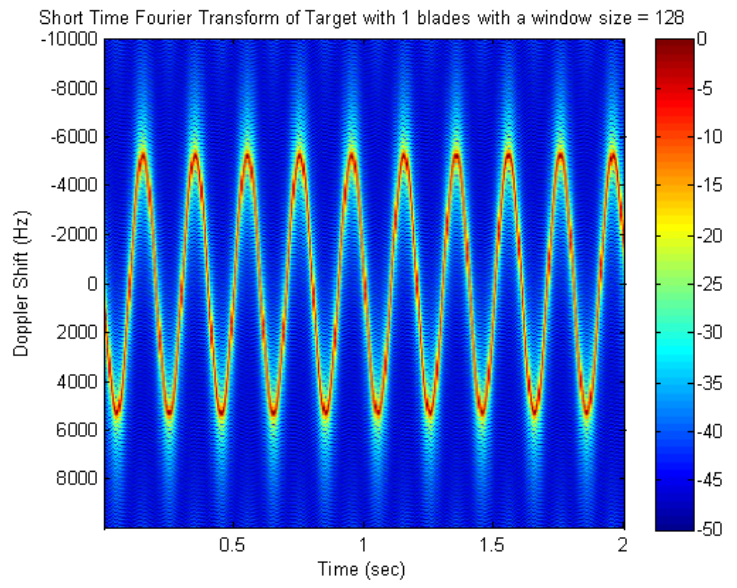

Fig. 1. STFT of the simulated one-point-target single bladed windmill rotating at an angular rotation speed of 300 RPM (Rotations Per Minute) with a blade length of $2.5 \mathrm{~m}$.
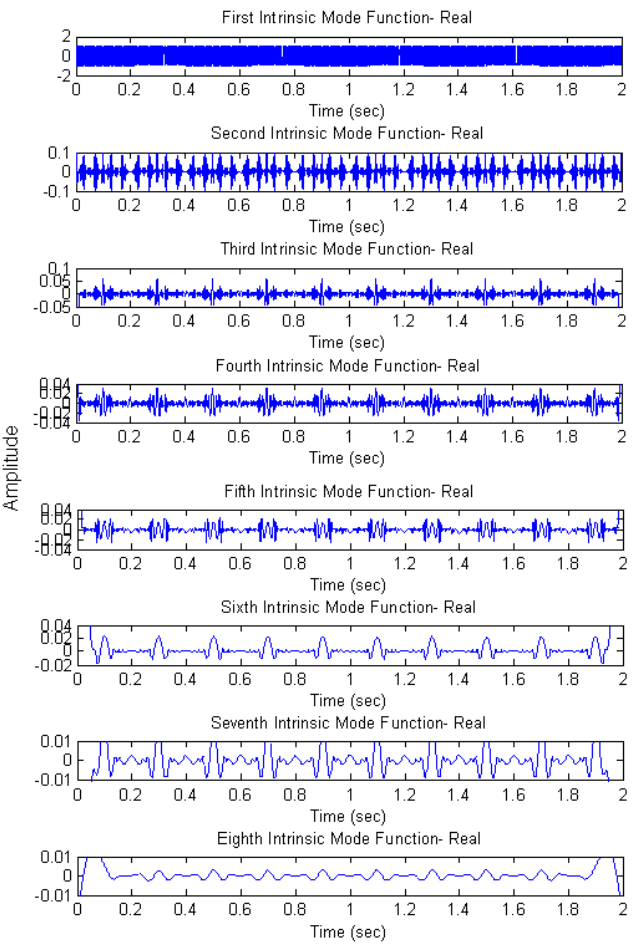

Fig. 2. The first 8 IMFs of the simulated one-point-target single bladed windmill rotating at an angular rotation speed of 300 RPM (Rotations Per Minute) with a blade length of $2.5 \mathrm{~m}$.

show that the extracted IMFs of order greater than 2, from IMF3 to IMF8, have a clear periodicity of 0.2 seconds which corresponds to the period of the rotating blade. IMF1 and IMF2 also present a periodic behavior but this is not visually evident due to the higher frequency components which are present in these signals.

In order to understand the effect of noise on the target echo IMFs, simulations were repeated by adding different levels of noise. To do this, white Gaussian noise was added to the simulated complex envelope of the received signal and then the EMD process applied. Simulations were repeated for SNR 


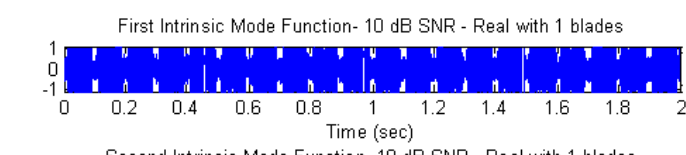

Second Intrinsic Mode Function- $10 \mathrm{~dB}$ SNR - Real with 1 blades

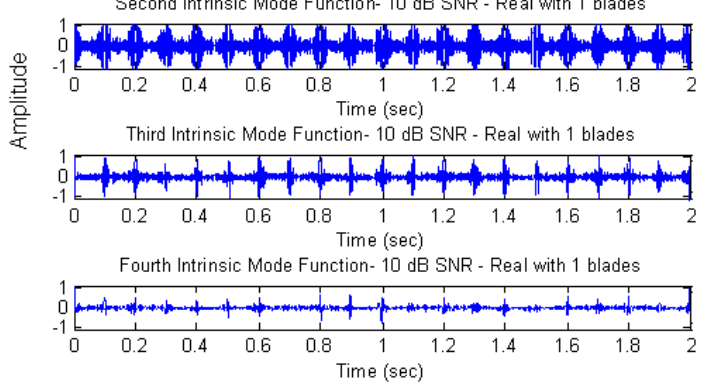

Fig. 3. The first 4 IMFs of the simulated one-point-target single bladed windmill rotating at an angular rotation speed of 300 RPM (Rotations Per Minute) with a blade length of $2.5 \mathrm{~m}$ for a Signal to Noise Ratio equal to 10 dB.

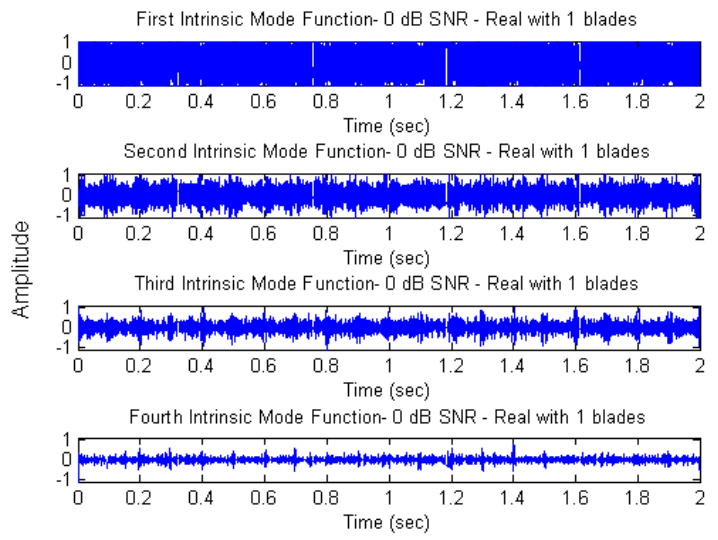

Fig. 4. The first 4 IMFs of the simulated one-point-target single bladed windmill rotating at an angular rotation speed of 300 RPM (Rotations Per Minute) with a blade length of $2.5 \mathrm{~m}$ for a Signal to Noise Ratio equal to 0 dB.

of $20 \mathrm{~dB}, 10 \mathrm{~dB}$ and $0 \mathrm{~dB}$. The first four IMFs extracted from the simulated data for a $10 \mathrm{~dB}$ and a $0 \mathrm{~dB}$ SNR are shown in Figure 3 and Figure 4, respectively. Results show that when the SNR decreases the first IMFs of the signal become increasingly corrupted, resulting in a loss of information. Results also show that the lower the SNR, the larger is the number of IMFs that become corrupted.

\section{EXPERIMENTAL SETUP}

Experimental testing was conducted with a windmill like target with a single blade to measure the return signal using a $\mathrm{CW}$ radar operating at $10 \mathrm{GHz}$. Echoes from the target were collected for an aspect angle of 0 degrees with respect to the radar as well as for an aspect angle of 45 degrees in order to increase the target reflecting area and enhance the target Radar Cross Section (RCS). The data was collected for 2 different rotation speeds that were controlled by the voltage applied to the blade system. The two voltages used were 7 Volts and 10 Volts, which resulted in a velocity of approximately 2 cycles/s $(2 \mathrm{~Hz})$ and 3.2 cycles/s $(3.2 \mathrm{~Hz})$, respectively. A TiePie oscilloscope was used to digitise the I and $\mathrm{Q}$ signal

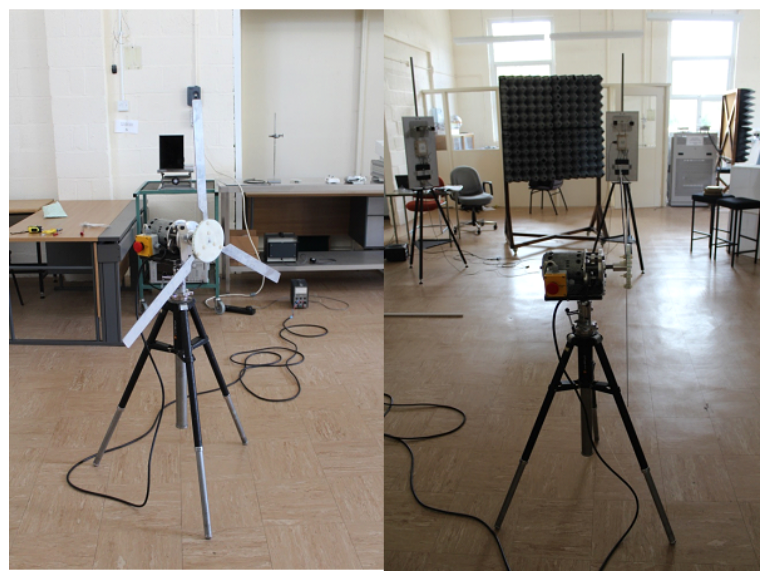

Fig. 5. Photos of the experimental setup. The CW radar was facing the rotating windmill like target.

components of the target echo. A sampling rate of $100 \mathrm{kHz}$ was used to collect 100000 samples at a time leading to a sequence of recordings of 1 second. The blade was $48 \mathrm{~cm}$ long from the centre point to the tip of the blade and was situated $330 \mathrm{~cm}$ from the radar. The setup for the experiment can be seen in Figure 5.

\section{Results}

The STFT of the complex signal of the echo from the single blade rotating with a 45 degree aspect angle and for an input voltage of 10 Volts $(3.2 \mathrm{~Hz})$ is shown in Figure 7 . This was obtained with a window size of 2000 samples, corresponding to an integration time of $20 \mathrm{~ms}$. Results clearly show the periodicity of the blades with a large contribution around the zero Doppler due to the white disc located at the centre of the windmill target. The graph in Figure 6 shows the real part of the complex signal for the same target. As the rotation speed is $3.2 \mathrm{~Hz}$, the expected periodicity is 0.32 seconds which can be visually seen in the plot, and which is particularly noticeable through the sections at 0.2-0.4 s and 0.6-0.8 s highlighted with the red circles. The IMFs of the rotating blade are shown in Figure 8. These were extracted from the in-phase component of the complex signal (the real part). Results show that the information on the target periodicity is not as visually evident as in the results obtained with the simulated data. However, the IMFs show a promising periodic trend, which is visible in IMF6, IMF7 and IMF8. This may be due to both the effect of noise and multipath as well to the rotation speed of the blade which was not $100 \%$ steady. Work is underway to clean the IMFs of unwanted returns and to extract the information on the periodicity of the target echo automatically.

Further experimental testing was conducted with a small helicopter model consisting of two $17.5 \mathrm{~cm}$ long blades as shown in Figure 9. The helicopter was placed in front of the radar at a distance of $306 \mathrm{~cm}$ and at the same height as the horn antennas. Measurements were taken for various rotation speeds. Unfortunately, it was not possible to measure the exact values of the rotation speeds nor this information was made available in the helicopter datasheet. The same TiePie oscilloscope was used to digitise the echoes from the moving helicopter with a sample rate of $100 \mathrm{kHz}$. Sequences 


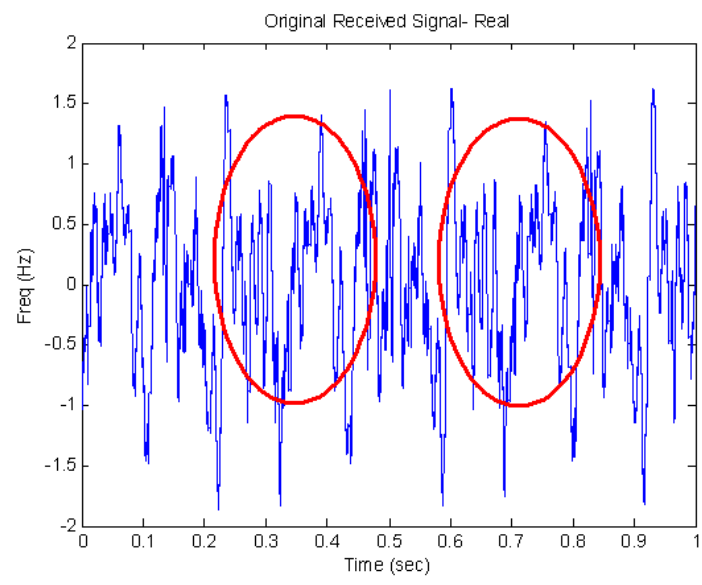

Fig. 6. The in-phase component of the experimental data for a single rotating blade. The aspect angle between the windmill target and the radar was 45 degree and the windmill was fed with 10 Volts corresponding to a rotation frequency of about $3.2 \mathrm{~Hz}$.

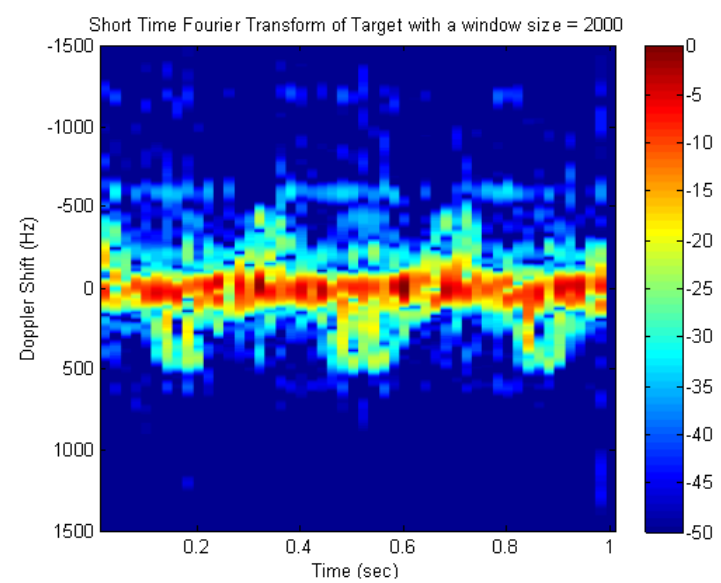

Fig. 7. The STFT of the experimental data for a single rotating blade. The aspect angle between the windmill target and the radar was 45 degree and the windmill was fed with 10 Volts corresponding to a rotation frequency of about $3.2 \mathrm{~Hz}$.

of recordings of $0.5 \mathrm{sec}$ were collected. The speed of the helicopters was controlled with a remote control provided with the helicopter. The STFT and the EMD process were applied to experimental data captured for the helicopter with the blades starting stationary, and progressing in speed until reaching full speed. This was done to investigate the effect of changing speed on the IMFs and to identify unambiguously which echo IMFs were carrying the information on the target. The STFT of the data is shown in Figure 10. It is evident that the frequency shift increases as the speed of the blades increases. A Hanning filter was applied to each window of the STFT to push down the side lobes. The number of elements of the window for these plots is 512, resulting in an integration time of $5.1 \mathrm{~ms}$ and in a frequency resolution of $196 \mathrm{~Hz}$. The EMD process was applied to the in-phase component of the data to produce the IMFs shown in Figure 11. Results clearly show that the IMF3 and IMF4 only start showing any information once the rotor blades begin to spin. Similar results were found for the IMFs of higher order. Interestingly, IMF1 and IMF2 do not show substantial variations for the entire time suggesting these are

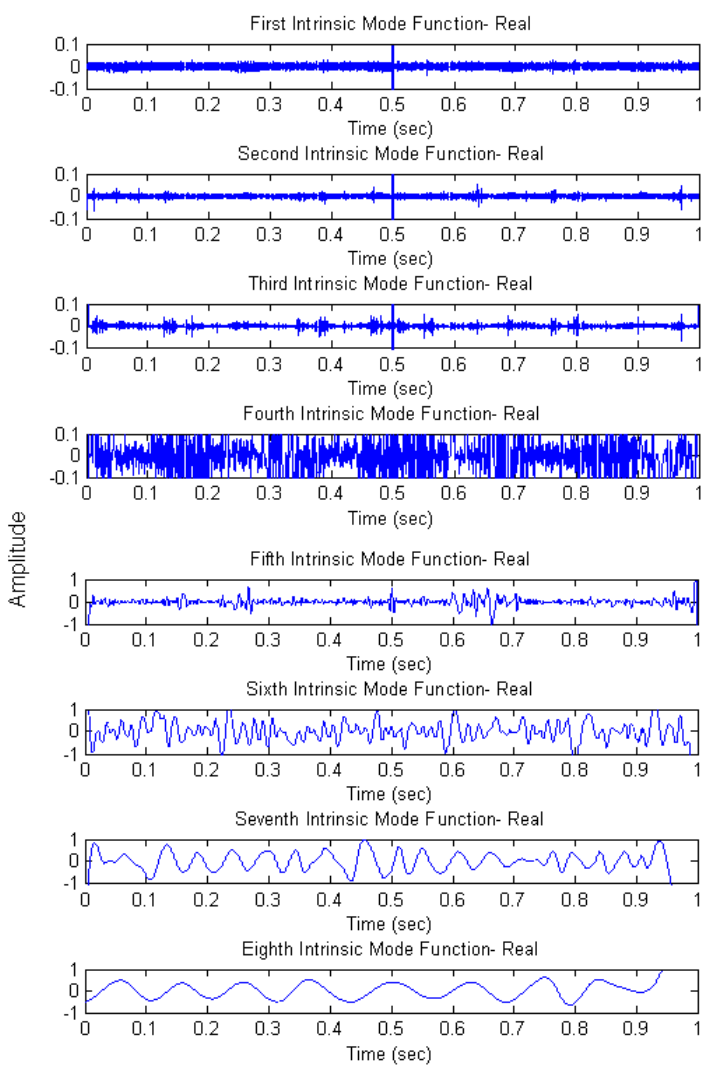

Fig. 8. The first 8 IMFs of the experimental data for a single rotating blade. The aspect angle between the windmill target and the radar was 45 degree and the windmill was fed with 10 Volts corresponding to a rotation frequency of about $3.2 \mathrm{~Hz}$.

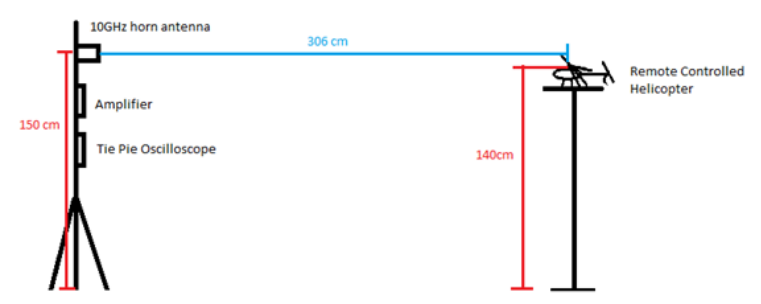

Fig. 9. A sketch of the experimental setup. The small helicopter was facing the $\mathrm{CW}$ radar at a distance of $306 \mathrm{~cm}$.

the IMFs carrying the noise and little, if any, information on the target. This is consistent with the properties of the IMFs with respect to noise as described in the simulations. The noise is characterised with a wider bandwidth than the micro-Doppler signature, and hence in a faster oscillation.

\section{CONCLUSION}

A set of experimental trials were conducted with a 10 $\mathrm{GHz} \mathrm{CW}$ radar to collect micro-Doppler signatures for a large single, double and triple bladed rotating fin and a sized miniature helicopters. The IMFs of the target echoes were extracted by using the EMD algorithm in order to investigate the information available in the IMFs and their physical significance. A set of simulations were carried out to assist in 


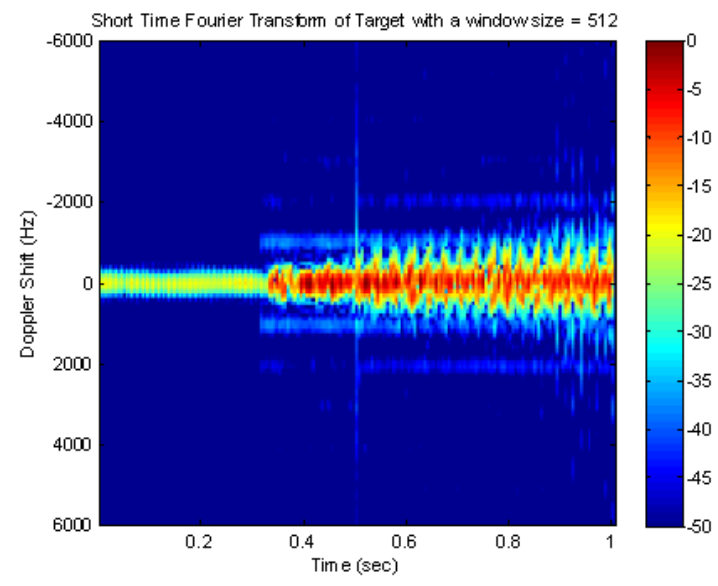

Fig. 10. Short Time Fourier Transform for the helicopter with the blades starting stationary, and progressing in speed until reaching full speed.

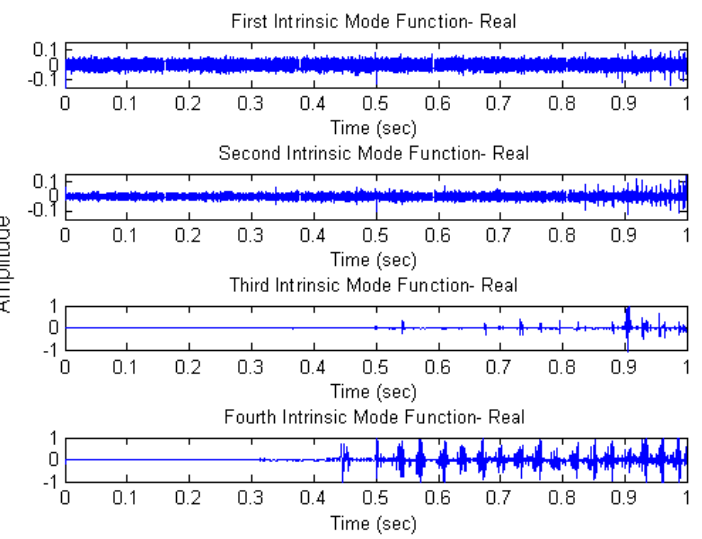

Fig. 11. The first 4 IMFs for the helicopter with the blades starting stationary, and progressing in speed until reaching full speed.

the interpretation of the experimental results and to investigate the effect of noise on the target IMFs. Results show the target IMFs carry information on the target and can be potentially used to extract features, such as blade rotation speed, to enable target classification.

\section{REFERENCES}

[1] V. Chen, F. Li, S. Ho, and H. Wechsler, "Micro-doppler effect in radar: phenomenon, model, and simulation study," IEEE Transactions on Aerospace and Electronic Systems, vol. 42, pp. 2-21, 2006.

[2] V. Chen, Micro-Doppler Effect in Radar. Artech House, Norwood, 2011.

[3] C. Clemente, A. Balleri, K. Woodbridge, and J. Soraghan, "Developments in target micro-doppler signatures analysis: radar imaging, ultrasound and through-the-wall radar," EURASIP Journal on Advances in Signal Processing, vol. 2013, no. 1, p. 47, 2013.

[4] V. C. Chen, D. Tahmoush, and W. J. Miceli, Radar Micro-Doppler Signatures: Processing and Applications, ser. Radar, Sonar, Navigation and Avionics. Institution of Engineering and Technology, 2014.

[5] T. Thayaparan, S. Abrol, E. Riseborough, L. Stankovic, D. Lamothe, and G. Duff, "Analysis of radar micro-doppler signatures from experimental helicopter and human data," IET Radar, Sonar and Navigation, vol. 1, no. 4, pp. 289-299, Aug 2007.

[6] P. Molchanov, K. Egiazarian, J. Astola, A. Totsky, S. Leshchenko, and M. Jarabo-Amores, "Classification of aircraft using micro-doppler bicoherence-based features," IEEE Transactions on Aerospace and Electronic Systems, vol. 50, no. 2, pp. 1455-1467, April 2014.

[7] P. Molchanov, A. Totsky, J. Astola, K. Egiazarian, S. Leshchenko, and M. Rosa-Zurera, "Aerial target classification by micro-doppler signatures and bicoherence-based features," in 9th European Radar Conference 2012 (EuRAD), Oct 2012, pp. 214-217.

[8] P. Setlur, F. Ahmad, and M. Amin, "Helicopter radar return analysis: Estimation and blade number selection," Signal Processing, vol. 91, no. 6, pp. 1409-1424, Jun. 2011.

[9] T. Hai-Tan, R. Melino, P. Berry, and D. Yau, "Microwave radar imaging of rotating blades," in 2013 International Conference on Radar (Radar), Sept 2013, pp. 202-207.

[10] H. Tran and R. Melino, "Application of the fractional fourier transform and s-method in doppler radar tomography," Electronic Warfare and Radar Division, Defence Science and Technology Organisation (DSTO), Australia, Tech. Rep. DSTO-RR-0357, 2010.

[11] A. Cilliers and W. Nel, "Helicopter parameter extraction using joint time-frequency and tomographic techniques," in 2008 International Conference on Radar, Sept 2008, pp. 598-603.

[12] N. E. Huang, Z. Shen, S. R. Long, M. C. Wu, H. H. Shih, Q. Zheng, N.C. Yen, C. C. Tung, and H. H. Liu, "The empirical mode decomposition and the hilbert spectrum for nonlinear and non-stationary time series analysis," Proceedings of the Royal Society of London A: Mathematical, Physical and Engineering Sciences, vol. 454, no. 1971, pp. 903-995, 1998.

[13] C.-P. Lai, R. Narayanan, Q. Ruan, and A. Davydov, "Hilbert-huang transform analysis of human activities using through-wall noise and noise-like radar," IET Radar, Sonar and Navigation, vol. 2, no. 4, pp. 244-25, August 2008.

[14] D. Fairchild and R. Narayanan, "Classification of human motions using empirical mode decomposition of human micro-doppler signatures," IET Radar, Sonar and Navigation, vol. 8, no. 5, pp. 425-434, June 2014.

[15] D. P. Fairchild and R. M. Narayanan, "Human activity classification using hilbert-huang transform analysis of radar doppler data," Proceedings of SPIE, Radar Sensor Technology XV, vol. 8021, June 2011.

[16] R. Narayanan, M. Shastry, P. Chen, and M. Levi, "Through-the-wall detection of stationary human targets using doppler radar," Progress In Electromagnetics Research B, vol. 20, pp. 147-166, 2010.

[17] P.-H. Chen, M. Shastry, C.-P. Lai, and R. Narayanan, "A portable realtime digital noise radar system for through-the-wall imaging," IEEE Transactions on Geoscience and Remote Sensing, vol. 50, no. 10, pp. 4123-4134, Oct 2012.

[18] J. Niu, Y. Liu, W. Jiang, X. Li, and G. Kuang, "Weighted average frequency algorithm for hilbert-huang spectrum and its application to micro-doppler estimation," IET Radar, Sonar and Navigation, vol. 6, no. 7, pp. 595-602, August 2012 